\title{
Resistencia a la compresión de la laja tipo salmón procedente de San José de Gracia, Molcaxac, Puebla, México*
}

\section{Adolfo Manuel Morales-Tassinari \\ Emanuel Gómez-Alonso \\ Moisés Zárate-Reyes}

Tecnológico Nacional de México

Instituto Tecnológico Superior de Tepexi de Rodríguez

Ingeniería Mecánica

Av. Tecnológico $s / n$., Col. Barrio San Sebastián Sección

Primera, CP. 74690, Tepexi de Rodríguez, Puebla.

MÉXICO

correo electrónico (email): tassinari.adolfo1973@gmail.com

Recibido 13-02-2020, aceptado 25-05-2020.

\section{Resumen}

El estudio tiene como propósito determinar la resistencia a la compresión de la laja tipo Salmón procedente de San José de Gracia, Molcaxac, Puebla, México. Para lograr esto, se desarrollaron ensayos de compresión uniaxial siguiendo el procedimiento descrito en la norma ASTM C170/C170M-16 Standard Test Method for Compressive Strength of Dimension Stone. La empresa Mármoles Gómez cortó 24 probetas cúbicas de esta piedra con dimensión de $50 \pm 0.5 \mathrm{~mm}$ en cada lado. Los ensayos se realizaron en el Laboratorio de Ciencias e Investigación en Materiales (LACIIM) del Centro de Competitividad y Tecnología para la Industria del Mármol del Estado de Puebla (CECOTIMEP) perteneciente al Instituto Tecnológico Superior de Tepexi de Rodríguez (ITSTR) del Tecnológico Nacional de México (TecNM). 12 probetas fueron secadas en horno a temperatura de $60^{\circ} \mathrm{C}$ durante 48 horas obteniendo peso seco constante; otras 12 probetas fueron saturadas en agua destilada a temperatura ambiente durante 48 horas obteniendo peso saturado constante. Se realizaron ensayos de compresión uniaxial en: 6 probetas secas con carga paralela a la veta, 6 probetas secas con carga perpendicular a la veta, 6 probetas saturadas con carga paralela a la veta y 6 probetas saturadas

*Este artículo es la versión extendida de los mejores trabajos del Congreso Nacional de Ingeniería Electromecánica y de Sistemas 2019 con carga perpendicular a la veta. La velocidad del ensayo de compresión uniaxial fue de $0.5 \mathrm{MPa} / \mathrm{s}$. Los resultados muestran que la laja tipo Salmón tiene una resistencia a la compresión promedio de $56.35 \mathrm{MPa}$. En conclusión, la laja tipo salmón de esta localidad es recomendada para usos como columnas para mesas, patas para sillas y pedestales para lavabos en casa-habitación.

Palabras clave: caracterización mecánica, columnas, laja tipo salmón, ensayo de compresión uniaxial, resistencia a la compresión.

\section{Abstract \\ (Compressive strength of the slate type Salmón from San José de Gracia, Molcaxac, Puebla, Mexico)}

This study has the purpose to determine the compressive strength of the slate rock type Salmón get from San José de Gracia, Molcaxac, Puebla, Mexico. In order to achieve this, uniaxial compression test were developed following the procedure described in of ASTM C170/C170M-16 Standard Test Method for Compressive Strength of Dimension Stone. The company Mármoles Gómez cut 24 cubic samples of this stone with a dimension of $50 \pm 0.5 \mathrm{~mm}$ on each side. The tests were carried out at the Laboratorio de Ciencias e Investigación en Materiales (LACIIM) of the Centro de Competitividad y Tecnología para la Industria del Mármol del Estado de Puebla (CECOTIMEP) belonging to the Instituto Tecnológico Superior de Tepexi de Rodríguez (ITSTR) of the Tecnológico Nacional de México (TecNM). 12 specimens were oven dried at a temperature of $60^{\circ} \mathrm{C}$ for 48 hours obtaining constant dry weight; another 12 specimens were saturated in distilled water at room temperature for 48 hours obtaining constant saturated weight. Uniaxial compression tests were performed: 6 dry specimens aligned parallel load to the vein, 6 dry specimens loaded perpendicular to the vein, 6 saturated specimens aligned parallel to the vein, and 6 saturated specimens loaded perpendicular to the vein. The speed of the uniaxial compression test was $0.5 \mathrm{MPa} / \mathrm{s}$. The result shows that the slate rock type salmón has an average compressive strength of 56.35 MPa. In conclusion, the slate rock type Salmón of this town is recommended for uses as columns for tables, legs for chairs and pedestals for washbowls in house-room.

Index terms: columns, compressive strength, mechanical characterization, slate rock type Salmon, uniaxial compression. 


\section{Introducción}

Las rocas son agregados naturales duros y compactos de partículas minerales con fuertes uniones cohesivas permanentes, habitualmente se consideran un sistema continuo [1]. Geológicamente, las rocas se catalogan en: a) ígneas, se producen cuando el magma se enfría y se solidifica; b) sedimentarias, constituidas por partículas y sedimentos a través de la meteorización de otras piedras, la aglomeración de material biológico, la precipitación de sustancias químicas o bioquímicas, o un conjunto de ellas; c) metamórficas, que experimentan modificaciones en su estructura y mineralogía atribuida a la temperatura y presión a grandes profundidades [2]. En este sentido, la laja o pizarra es una roca metamórfica microcristalina comúnmente derivada del esquisto y compuesta por mica, clorita y cuarzo; así, los minerales micáceos tienen una orientación subparalela y, por lo tanto, imparten una fuerte escisión a la roca que permite que esta última se divida en láminas delgadas pero resistentes [3].

La laja se origina casi siempre por el metamorfismo en grado bajo de lutitas y pelitas; con menor frecuencia, producidas por el metamorfismo de las cenizas volcánicas [4]. La laja contiene una textura foliada densa $y$, como esta roca se divide a lo largo de la dirección de la escisión pizarrosa, debilita su resistencia a la erosión y a la intemperie [5]. Así, la laja de grado arquitectónico debe de estar libre de grietas, costuras $\mathrm{u}$ otros rasgos que puedan afectar su integridad estructural o función; además, las variaciones características de color inherentes de la cantera de la que se obtiene son aceptables [6]. Las explotaciones de la laja se realizan a cielo abierto mediante desmonte con maquinaría, perforación y voladura; de esta

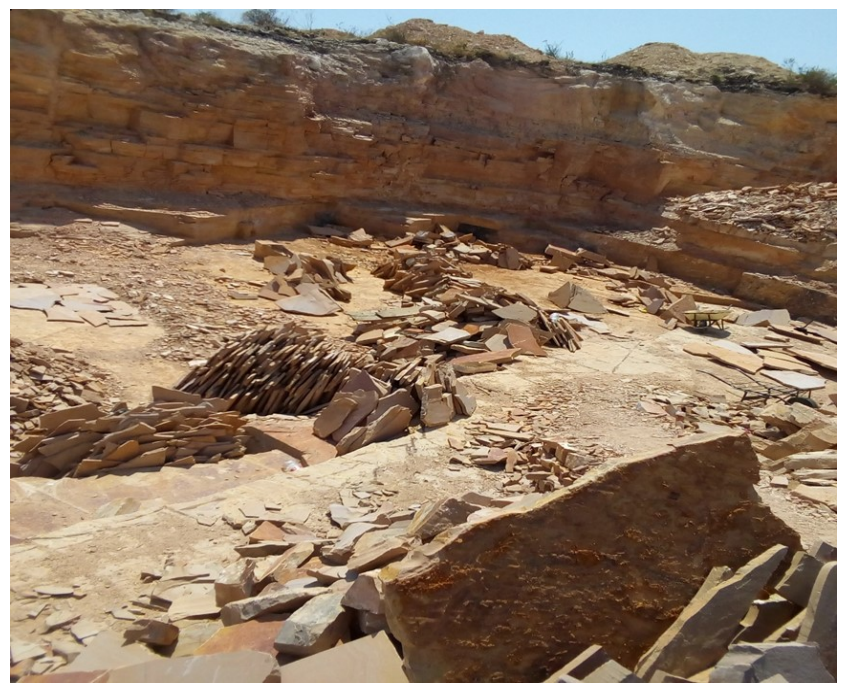

Fig. 1. Cantera de laja tipo salmón.

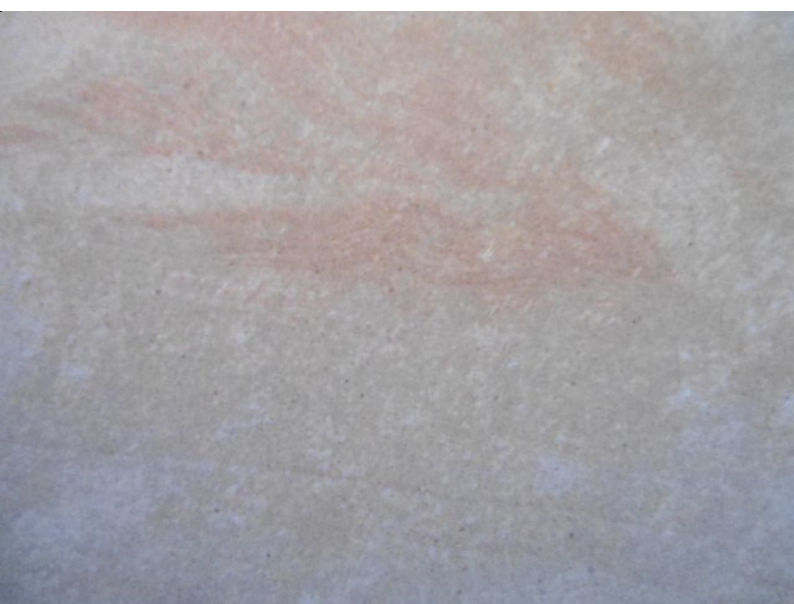

Fig. 2. Superficie de la laja tipo salmón.

forma, la laja se separa con cuñas y otras herramientas clasificándose por tamaños, grosores y, en ciertos casos, se cortan y dimensionan empleando discos diamantados [7]. Los colores de la laja son variados desde el gris y ocre hasta el marrón; además, de colores azules, rojos, negros y verdes, según la incidencia de óxidos de fierro, manganeso y otros minerales.

En la localidad de San José de Gracia, Molcaxac, Puebla, México, existen canteras de laja tipo Salmón que es una roca de color salmón con vetas obscuras, y que se comercializan para fachadas exteriores. En la Fig. 1 se observa una cantera de laja tipo Salmón en dicha localidad; mientras que en la Fig. 2 se observa el aspecto superficial de la laja tipo salmón.

Los ensayos de caracterización evalúan la ventaja que tiene una roca para una aplicación específica [8]; por ello, para obtener las características mecánicas de una roca se caracterizan el comportamiento mecánico y de deformaciones, donde estas propiedades mecánicas permiten representar la resistencia a: tensión, compresión, flexión y choque [9]. En este sentido, la resistencia a la compresión en una roca establece la fuerza a compresión máxima que resiste el material sin aplastarse ni deformarse más de lo permitido [8]; por ello, una prueba muy empleada en laboratorio para obtener la resistencia a la compresión es la prueba de compresión uniaxial, que se basa en emplear una fuerza de manera axial y a compresión a través de una máquina sobre un área específica de una probeta cúbica o cilíndrica.

Diversos estudios internacionales han determinado la resistencia a la compresión de diversos tipos de rocas [10], [11], [12], [13], [14], [15]; cabe mencionar que, el conocimiento de esta resistencia a la compresión le otorga ventaja a la indus- 
tria de la construcción para el cumplimiento de estándares. Además, en México también se han llevado a cabo estudios para determinar la resistencia a la compresión de diversas rocas [16], [17], [18], [19], [20], [21]. En la Tabla 1, se muestran los valores de la resistencia a la compresión de diversos tipos de rocas internacionales y nacionales.

De manera que este artículo tiene la finalidad de presentar los resultados de la resistencia a la compresión de la laja tipo salmón de la localidad de San José de Gracia, Molcaxac, Puebla, México, a través de la prueba de compresión uniaxial aplicando la metodología de la norma ASTM C170/C170M-16 Standard Test Method for Compressive Strength, la cual consiste en realizar ensayos con cargas paralelas a la veta y perpendicular a la veta tanto en condición seca como en condición saturada [22]. Cabe aclarar que, la norma ASTM C170/C170M-16 señala las condiciones requeridas para realizar un ensayo de compresión uniaxial en condición seca y saturada con carga perpendicular y paralela a la veta, contando con al menos una muestra de un mínimo de 20 probetas. En este sentido, para los ensayos a compresión uniaxial se utilizaron 24 probetas cúbicas con dimensión de $50 \pm 0.5 \mathrm{~mm}$ en cada lado.

\section{Desarrollo}

Para la realización de los ensayos de compresión uniaxial se emplearon los equipos y materiales: pulidora marca MTI modelo UNIPOL-820, vernier digital marca POINT modelo DC-KH6, escuadra metálica de $90^{\circ}$ marca MITUTOYO, horno de secado marca MEMMERT modelo UP500, báscula digital marca METTLER TOLEDO modelo SP30002-S, máquina de compresión marca MATEST, termómetro digital marca MATEST, agua destilada marca HERCOTEC y lijas con tamaño de grano 400.

\subsection{Metodología de preparación de probetas}

Las muestras de la laja tipo salmón se obtuvieron de diversas rocas obtenidas de la cantera ubicada en la localidad de San José de Gracia, Molcaxac, Puebla, México. De las piedras obtenidas, se procesaron un total de 60 probetas; sin embargo, se seleccionaron un grupo de 24 probetas basado en la homogeneidad del color salmón y que la dimensión de cada probeta fuera de $50 \pm 0.5 \mathrm{~mm}$ en cada cara. Para la preparación de las probetas se siguió el procedimiento mostrado en el diagrama de flujo de la Fig. 3.

Tabla 1. Resistencia a la compresión de diversos tipos de rocas.

\begin{tabular}{|c|c|c|c|c|c|}
\hline \multicolumn{3}{|c|}{ Rocas Internacionales } & \multicolumn{3}{|c|}{ Rocas Nacionales } \\
\hline Lugar & Tipo de Roca & $\begin{array}{c}\text { Resistencia a la } \\
\text { Compresión } \\
\text { (MPa) }\end{array}$ & Lugar & Tipo de Roca & $\begin{array}{c}\text { Resistencia a la } \\
\text { Compresión } \\
\text { (MPa) }\end{array}$ \\
\hline Mahallat, Irán & $\begin{array}{c}\text { Travertino tipo } \\
\text { Atashkooh White } \\
{[10]}\end{array}$ & 49.3 & Yucatán & Roca caliza [16] & $27.72 \pm 15.8$ \\
\hline Asturias, España & $\begin{array}{c}\text { Piedra caliza tipo } \\
\text { Rojo Cornellana } \\
{[11]}\end{array}$ & 129.45 & $\begin{array}{c}\text { Cerro de la } \\
\text { Tambora de la } \\
\text { comunidad de El } \\
\text { Picacho, Tierra } \\
\text { Blanca, } \\
\text { Guanajuato }\end{array}$ & Toba riolítica [17] & 15.49 \\
\hline $\begin{array}{c}\text { Divčane, Jajce, } \\
\text { Bosnia- } \\
\text { Herzegovina }\end{array}$ & $\begin{array}{l}\text { Travertino tipo } \\
\text { Plivit Jajce [12] }\end{array}$ & 46 & $\begin{array}{l}\text { Guangochillo, } \\
\text { Barrial de } \\
\text { Guadalupe, } \\
\text { Torreón, } \\
\text { Coahuila }\end{array}$ & Mármol [18] & 59 \\
\hline $\begin{array}{c}\text { Callejón de } \\
\text { Huaylas, Áncash, } \\
\text { Perú }\end{array}$ & Granito [13] & 150.57 & $\begin{array}{c}\text { Moralillo, Tepexi } \\
\text { de Rodríguez, } \\
\text { Puebla }\end{array}$ & $\begin{array}{l}\text { Travertino tipo } \\
\text { Puebla [19] }\end{array}$ & 64.51 \\
\hline $\begin{array}{l}\text { Córdoba, } \\
\text { Colombia }\end{array}$ & $\begin{array}{c}\text { Caliza tipo } \\
\text { Mármol Royal } \\
\text { Bronce [14] }\end{array}$ & 45.54 & $\begin{array}{c}\text { El Rosario } \\
\text { Xochitiopan, } \\
\text { Zacapala, Puebla }\end{array}$ & Roca caliza [20] & 12 \\
\hline $\begin{array}{l}\text { Cojitambo, Cañar, } \\
\text { Ecuador }\end{array}$ & $\begin{array}{l}\text { Roca del } \\
\text { afloramiento [15] }\end{array}$ & 23 a 32 & $\begin{array}{l}\text { Ixcaquixtla, } \\
\text { Puebla }\end{array}$ & $\begin{array}{c}\text { Travertino tipo } \\
\text { Dorado Tepexi } \\
\qquad[21\rceil\end{array}$ & 66 \\
\hline
\end{tabular}




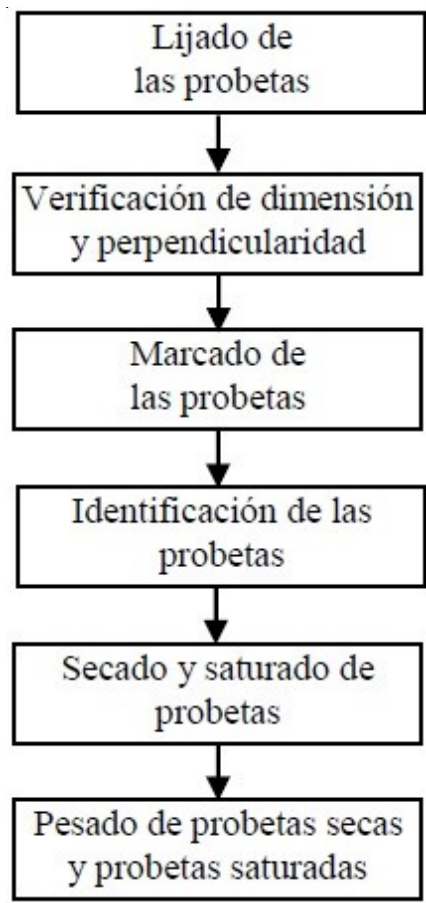

Fig. 3. Diagrama de flujo de la preparación de las probetas.

Para la preparación, las 24 probetas fueron lijadas en las caras que tuvieran contacto con los dados del equipo de compresión, con la finalidad de que el contacto entre los dados y las probetas fuera lo más uniformes posibles. Durante el proceso de lijado, se verificó con una escuadra metálica que las caras estuvieran planas y que fueran perpendiculares. En la Fig. 4a), se muestra el lijado de una probeta; mientras que en la Fig. 4b), se observa la verificación de una probeta.
Posterior de lijar con grano 400 las 24 probetas en las dos caras, se marcó aleatoriamente la orientación de carga para facilitar su colocación en la máquina de compresión; por lo que, 12 probetas fueron marcadas con una línea paralela a la veta y 12 probetas fueron marcadas con una línea perpendicular a la veta. Además, se estableció un número de identificación en cada probeta para distinguirlas durante las pruebas de compresión uniaxial. En la Fig. 5a), se observan las marcas de orientación de carga; mientras que en la Fig. 5b), se observan las probetas numeradas y marcadas.

Previo al ensayo de compresión uniaxial y con base en la norma ASTM C170/C170M-16, 12 especímenes fueron secadas en el horno durante 48 horas a una temperatura de $60 \pm 2^{\circ} \mathrm{C}$; al mismo tiempo, 12 especímenes fueron sumergidas en agua destilada durante 48 horas a temperatura ambiente. En la Fig. 6a), se observan los especímenes en el horno; mientras que en la Fig. 6b), se observan los especímenes sumergidos en agua destilada.

Cabe mencionar que tanto para el secado y la saturado solo se consideró una variable que es el contenido de humedad [23]. Para garantizar que las 12 probetas dentro del horno estuvieran secas, fueron pesadas tres veces previo a su salida del horno mostrando en estas tres horas consecutivas ningún cambio significativo en su peso. También, las 12 probetas sumergidas en agua destilada fueron pesadas tres veces previo a su salida de inmersión no mostrando cambio significativo en su peso. Cabe aclarar que, las probetas secas y saturadas fueron pesadas a temperatura ambiente promedio de $22^{\circ} \mathrm{C}$. En la Tabla 3, se muestran los valores del peso seco del grupo de probetas PA y PB; además, se muestran los valores de los pesos saturados del grupo de probetas PC y PD.
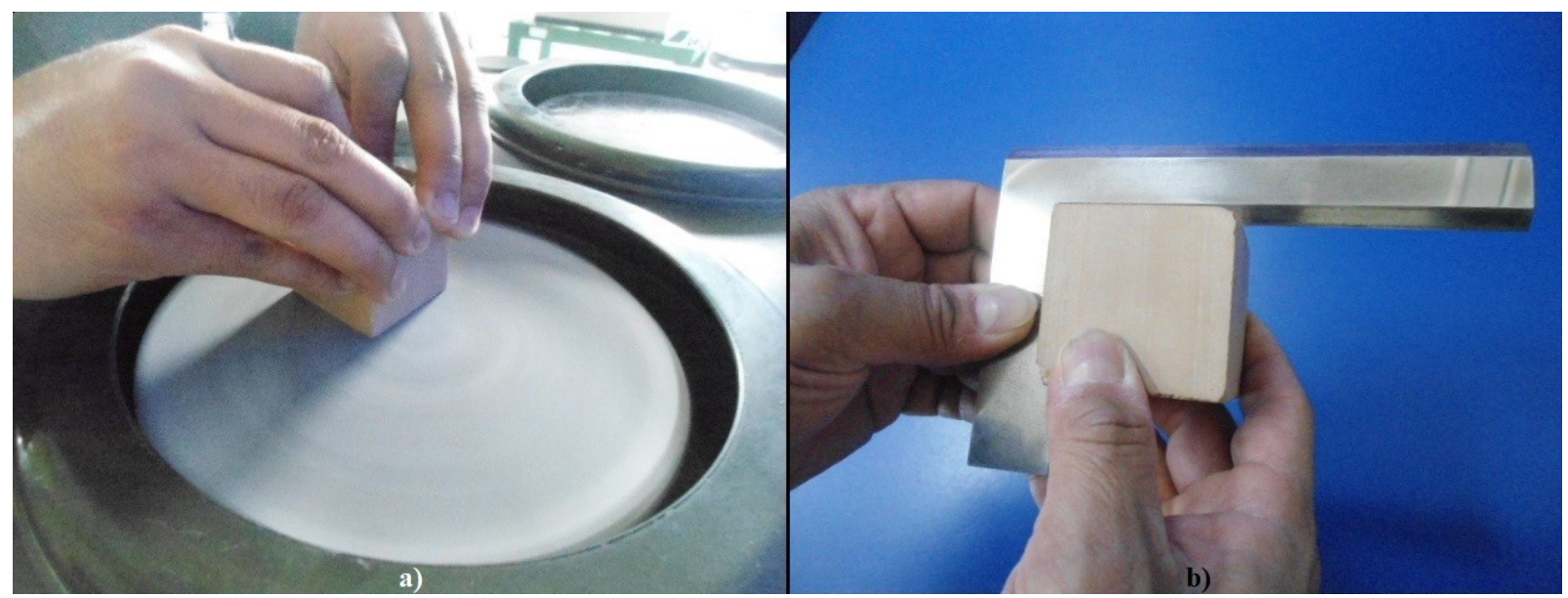

Fig. 4. a) Lijado de una probeta; b) Verificación de una probeta. 


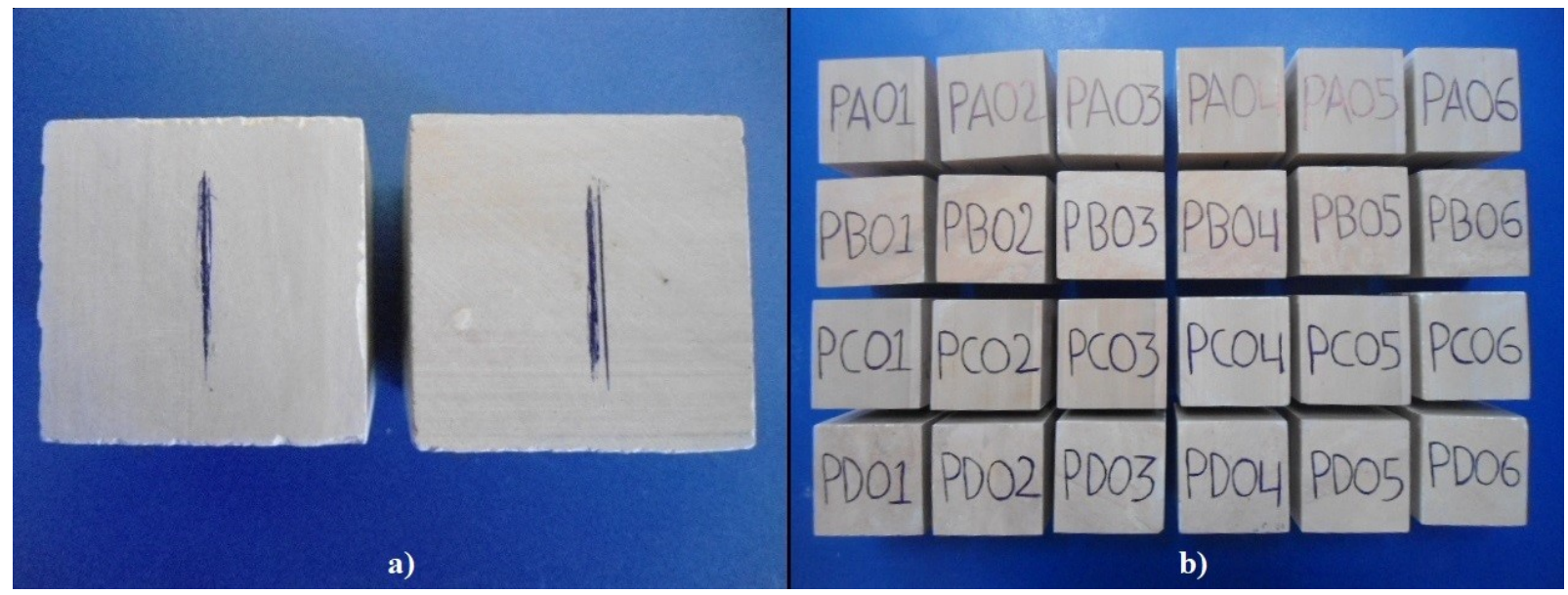

Fig. 5. a) Marca de orientación de carga en dos probetas; b) Probetas numeradas y marcadas.

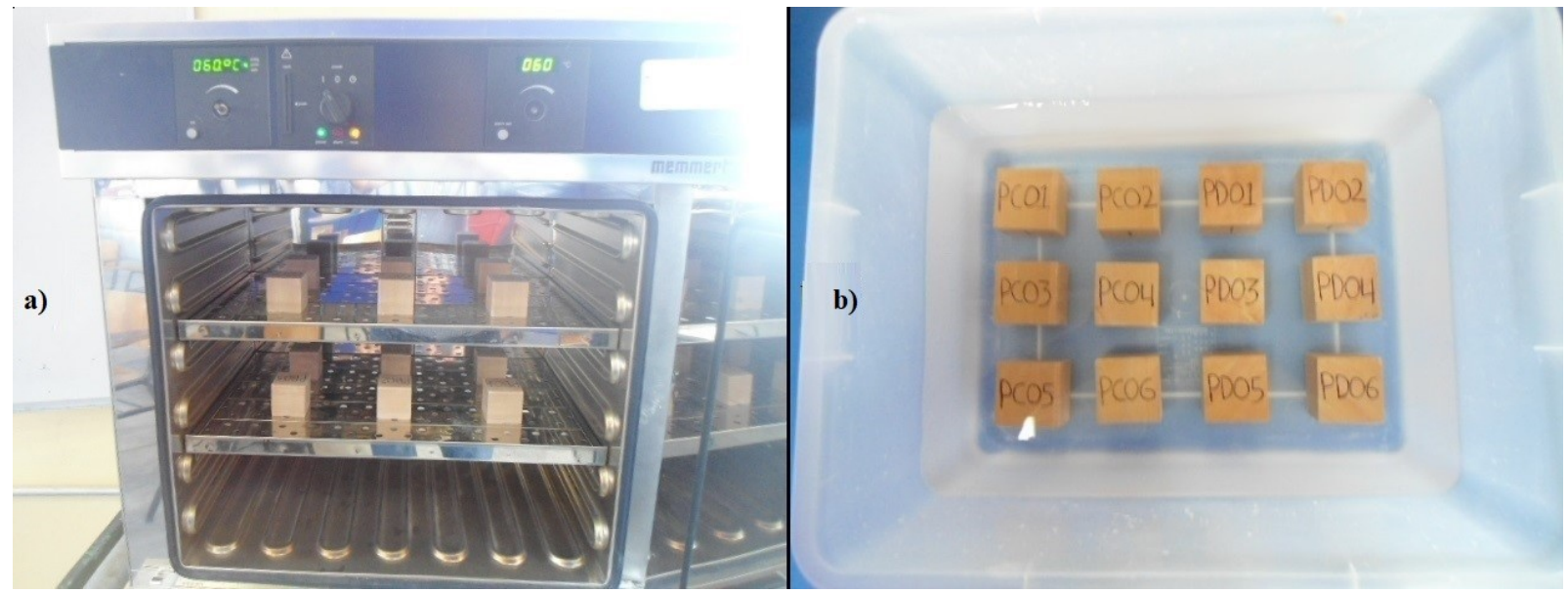

Fig. 6. a) 12 especímenes dentro del horno de secado; b) 12 especímenes sumergidos en agua destilada.

\subsection{Ensayo de compresión uniaxial de probetas secas y probetas saturadas}

Las probetas se colocaron en el equipo de compresión de manera que quedaran centradas en los dados y que la orientación de carga quedara al frente para poderla visualizar; además, con base en la norma ASTM C170/C170M-16, la velocidad de aplicación de carga fue de $0.5 \mathrm{MPa} / \mathrm{s}$ [22]. En la Fig. 7, se observa una probeta colocada en la máquina de compresión.

También como la misma norma señala, se realizaron ensayos de compresión uniaxial en 6 probetas secas con carga paralela a la veta, 6 probetas secas con carga perpendicular a la veta, 6 probetas saturadas con carga paralela a la veta y finalmente 6 probetas saturadas con carga perpendicular a la veta.
Tabla 3. Peso seco de las probetas PA y PB y peso saturado de las probetas PC y PD.

\begin{tabular}{cccc}
\hline $\begin{array}{c}\text { Número } \\
\text { de } \\
\text { Probeta }\end{array}$ & $\begin{array}{c}\text { Peso } \\
\text { Seco } \\
\mathbf{( g )}\end{array}$ & $\begin{array}{c}\text { Número } \\
\text { de } \\
\text { Probeta }\end{array}$ & $\begin{array}{c}\text { Peso } \\
\text { Saturado } \\
\mathbf{( g )}\end{array}$ \\
PA01 & 302.17 & PC01 & 315.12 \\
PA02 & 305.25 & PC02 & 316.69 \\
PA03 & 295.79 & PC03 & 311.61 \\
PA04 & 301.80 & PC04 & 312.94 \\
PA05 & 302.90 & PC05 & 313.70 \\
PA06 & 300.39 & PC06 & 311.78 \\
PB01 & 303.50 & PD01 & 316.46 \\
PB02 & 300.15 & PD02 & 311.22 \\
PB03 & 296.83 & PD03 & 308.00 \\
PB04 & 301.79 & PD04 & 310.98 \\
PB05 & 306.81 & PD05 & 310.97 \\
PB06 & 301.43 & PD06 & 307.11 \\
\hline
\end{tabular}




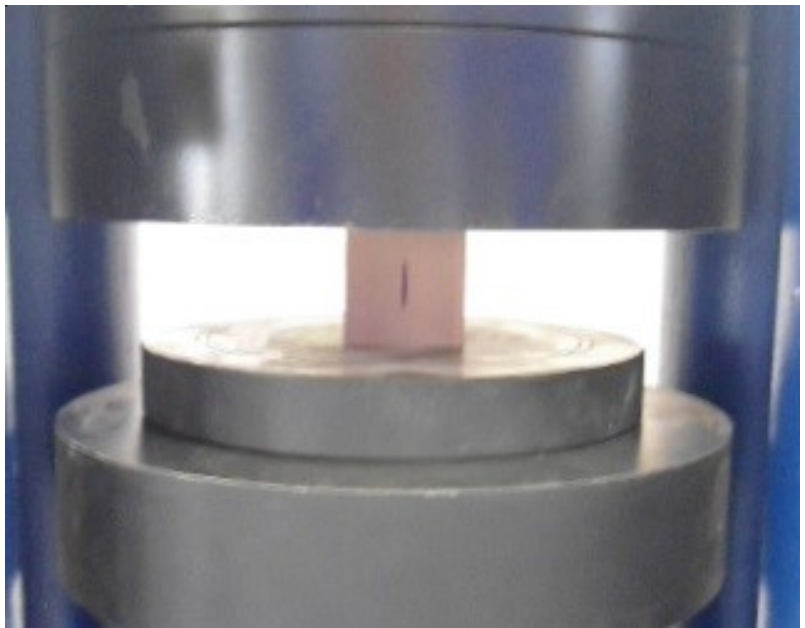

Fig. 7. Probeta colocada en la máquina de compresión
Siguiendo esta recomendación, las probetas PA01 a PA06 se probaron en condición seca con carga paralela a la veta, las probetas PB01 a PB06 se probaron en condición seca con carga perpendicular a la veta, las probetas PC01 a PC06 se probaron en condición saturada con carga paralela a la veta y que las probetas PD01 a PD06 se probaron en condición saturada con carga perpendicular a la veta. En la Fig. 8, se observan las 24 probetas de laja tipo Salmón después del ensayo de compresión uniaxial.

En las Figs. 9a) y 9c) se muestra que debido a que la carga de compresión era paralela a la veta, las rupturas de estas probetas ocurrieron orientadas a las vetas; por otro lado, en las Figs. 9b) y 9d) se observa que debido a que la carga de compresión era perpendicular a la veta, las rupturas de estas probetas ocurrieron sin orientación a las vetas.

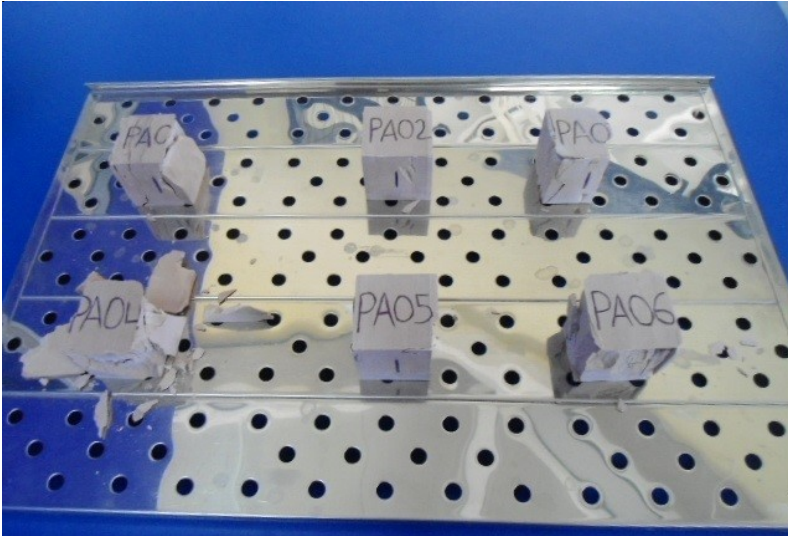

a)Probetas secas-paralelas

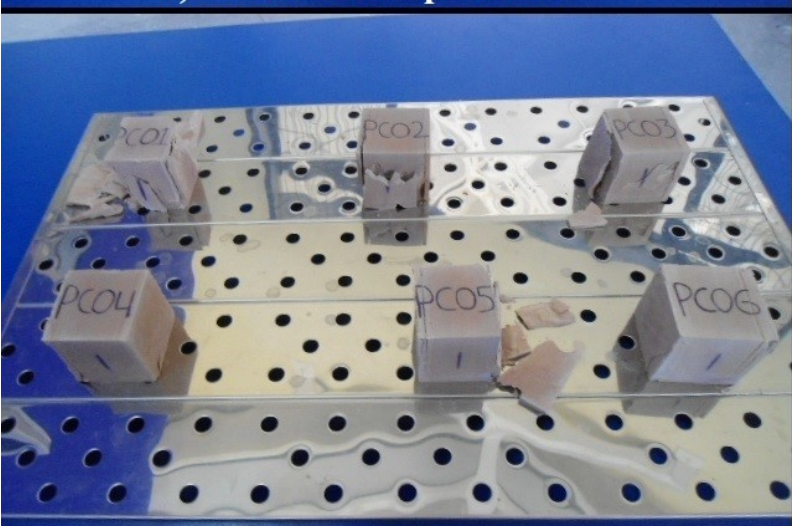

c) Probetas saturadas-paralelas

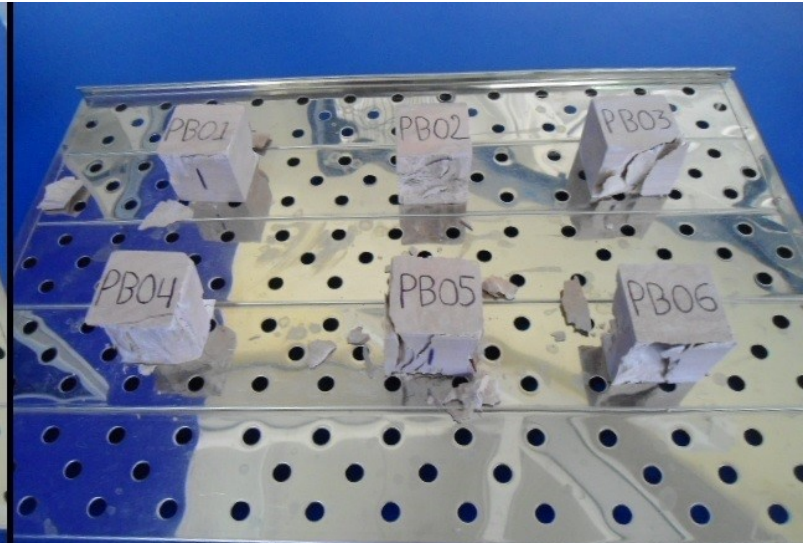

b) Probetas secas-perpendiculares

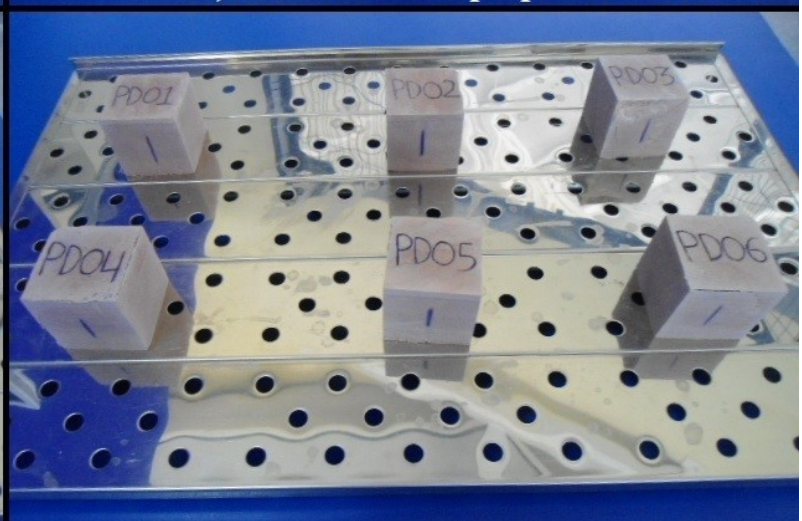

d) Probetas saturadas-perpendiculares

Fig. 8. Probetas después del ensayo de compresión uniaxial: a) Probetas secas-paralelas PA01-PA06; b) Probetas secas-perpendiculares PB01-PB06; c) Probetas saturadas-paralelas PC01-PC06; d) Probetas saturadas-perpendiculares PD01-PD06. 


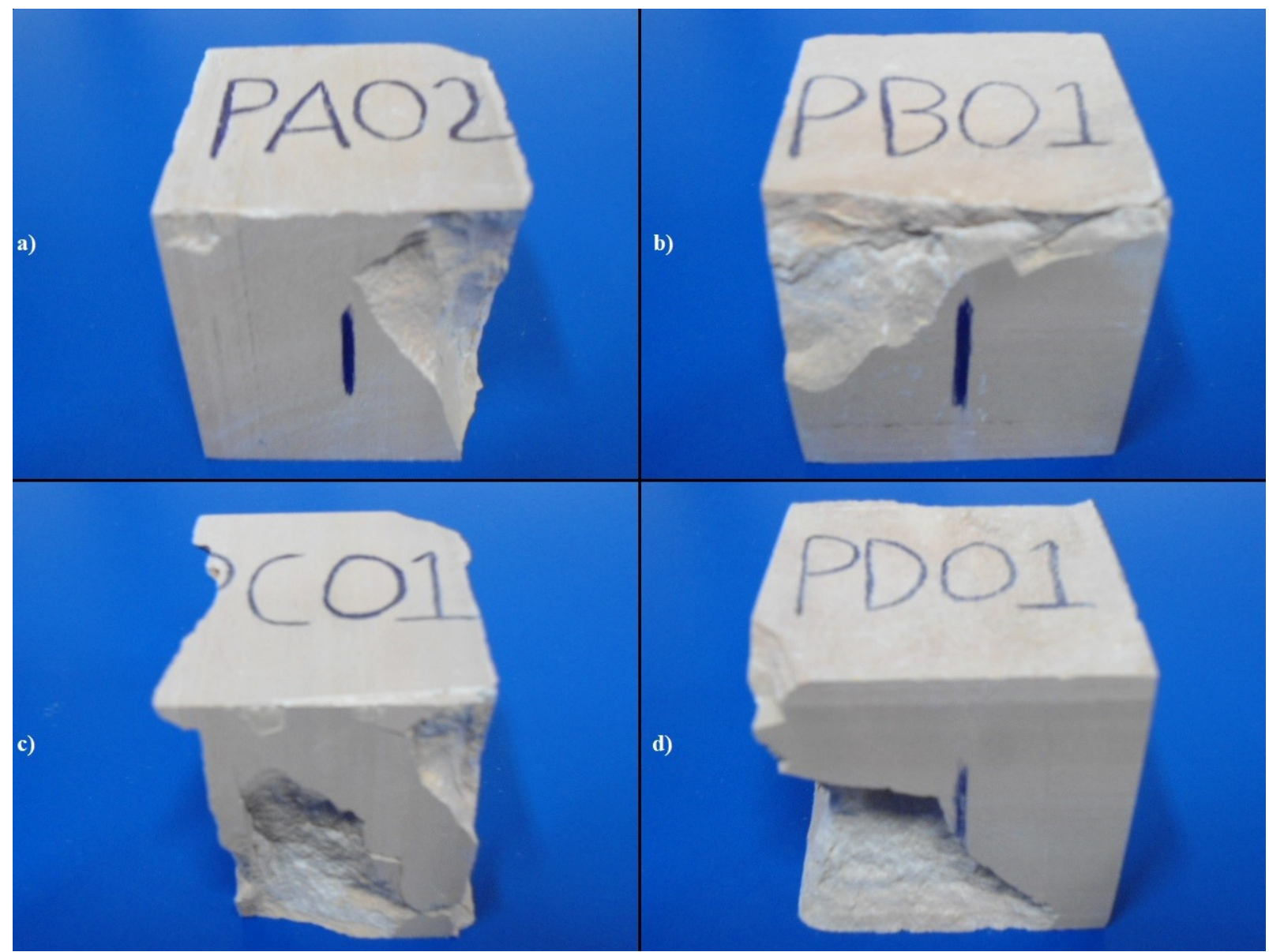

Fig. 9. a) Fotografía de la probeta PA02; b) Fotografía de la probeta PB01; c) Fotografía de la probeta PC01; d) Fotografía de la probeta PD01.

\section{Resultados}

Conforme a lo sugerido en la norma ASTM C170/C170M-16, se tomó la lectura de la carga de compresión axial $(W)$ a la cual las probetas entraron en ruptura y considerando el área efectiva de contacto de carga $\left(A_{C}\right)$ sobre la probeta se calculó la resistencia a la compresión $(S)$ aplicando la ecuación 1 [22].

$$
S=\frac{W}{A_{C}}
$$

En la Tabla 4, se observan los resultados obtenidos de la resistencia a la compresión de las 24 probetas de laja tipo salmón ensayadas.

Como se señala en los valores numéricos de la Tabla 4, las probetas secas con carga paralela a la veta tuvieron una resistencia la compresión promedio de $123.15 \mathrm{MPa}$ con una desviación estándar de $7.49 \mathrm{MPa}$, las probetas secas con carga perpendicular a la veta tuvieron una resistencia a la compresión promedio de $117.01 \mathrm{MPa}$ con una desviación estándar de $13.47 \mathrm{MPa}$, las probetas saturadas con carga paralela a la veta tuvieron una resistencia a la compresión promedio de $62.48 \mathrm{MPa}$ con una desviación estándar de $10.93 \mathrm{MPa}$, y las probetas saturadas con carga perpendicular a la veta tuvieron una resistencia a la compresión promedio de $56.35 \mathrm{MPa}$ con una desviación estándar de 5.23 MPa. En la Fig. 10, se observa el diagrama de caja comparativo de la resistencia a la compresión.

Como se observa en el diagrama de la Fig. 10, el grupo de probetas saturadas con carga perpendicular a la veta tuvo la resistencia a la compresión más baja; así, para saber si este valor es significativo se realizó un análisis de varianza de dos factores. Los factores que se consideraron fueron las condiciones físicas (probetas secas y probetas saturadas) y las 
Tabla 4. Resultados de la resistencia a la compresión.

\begin{tabular}{|c|c|c|c|c|}
\hline $\begin{array}{c}\text { Condición } \\
\text { de la } \\
\text { probeta }\end{array}$ & $\begin{array}{l}\text { Número } \\
\text { de } \\
\text { Probeta }\end{array}$ & $\begin{array}{c}\text { Carga } \\
\text { de } \\
\text { Compresión } \\
W(N)\end{array}$ & 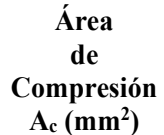 & $\begin{array}{c}\text { Resistencia a } \\
\text { la } \\
\text { Compresión } \\
\text { S (MPa) }\end{array}$ \\
\hline \multirow{8}{*}{$\begin{array}{l}\text { Probeta seca } \\
\text { con carga } \\
\text { paralela a la } \\
\text { veta }\end{array}$} & PA01 & 282622 & 2500.00 & 113.05 \\
\hline & PA02 & 331151 & 2520.03 & 131.41 \\
\hline & PA03 & 295137 & 2499.96 & 118.06 \\
\hline & PA04 & 325793 & 2510.00 & 129.80 \\
\hline & PA05 & 322649 & 2525.00 & 127.78 \\
\hline & PA06 & 298212 & 2510.00 & 118.81 \\
\hline & \multirow{2}{*}{\multicolumn{3}{|c|}{$\begin{array}{c}\text { Promedio } \\
\text { Desviación Estándar }\end{array}$}} & 123.15 \\
\hline & & & & 7.49 \\
\hline \multirow{8}{*}{$\begin{array}{l}\text { Probeta seca } \\
\text { con carga } \\
\text { perpendicular } \\
\text { a la veta }\end{array}$} & PB01 & 311218 & 2530.08 & 123.01 \\
\hline & PB02 & 307146 & 2504.98 & 122.61 \\
\hline & PB03 & 225125 & 2504.94 & 89.87 \\
\hline & PB04 & 311932 & 2515.00 & 124.03 \\
\hline & PB05 & 301573 & 2550.25 & 118.25 \\
\hline & PB06 & 312576 & 2514.90 & 124.29 \\
\hline & \multirow{2}{*}{\multicolumn{3}{|c|}{$\begin{array}{c}\text { Promedio } \\
\text { Desviación Estándar }\end{array}$}} & 117.01 \\
\hline & & & & 13.47 \\
\hline \multirow{8}{*}{$\begin{array}{c}\text { Probeta } \\
\text { saturada con } \\
\text { carga paralela } \\
\text { a la veta }\end{array}$} & PC01 & 191013 & 2540.16 & 75.20 \\
\hline & PC02 & 173921 & 2525.00 & 68.88 \\
\hline & $\mathrm{PC} 03$ & 183862 & 2535.10 & 72.53 \\
\hline & PC04 & 126722 & 2510.01 & 50.49 \\
\hline & PC05 & 138594 & 2520.00 & 55.00 \\
\hline & PC06 & 132300 & 2504.94 & 52.82 \\
\hline & \multirow{2}{*}{\multicolumn{3}{|c|}{$\begin{array}{c}\text { Promedio } \\
\text { Desviación Estándar }\end{array}$}} & 62.48 \\
\hline & & & & 10.93 \\
\hline \multirow{8}{*}{$\begin{array}{c}\text { Probeta } \\
\text { saturada con } \\
\text { carga } \\
\text { perpendicular } \\
\text { a la veta }\end{array}$} & PD01 & 156544 & 2540.16 & 61.63 \\
\hline & PD02 & 143671 & 2520.03 & 57.01 \\
\hline & PD03 & 131800 & 2485.02 & 53.04 \\
\hline & PD04 & 127867 & 2499.99 & 51.15 \\
\hline & PD05 & 158260 & 2494.80 & 63.44 \\
\hline & PD06 & 128868 & 2485.02 & 51.86 \\
\hline & \multirow{2}{*}{\multicolumn{3}{|c|}{$\begin{array}{c}\text { Promedio } \\
\text { Desviación Estándar }\end{array}$}} & 56.35 \\
\hline & & & & 5.23 \\
\hline
\end{tabular}

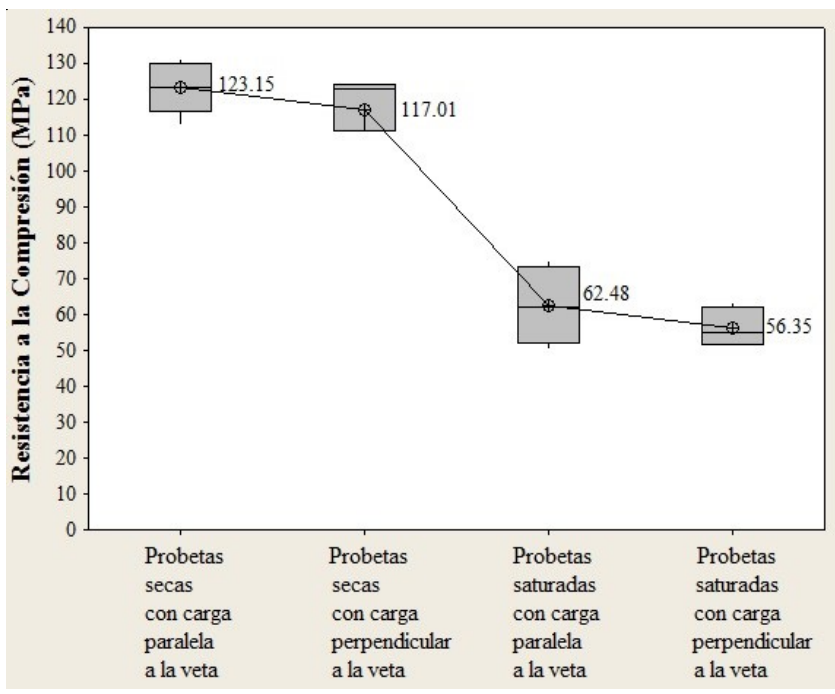

Fig. 10. Diagrama de caja comparativo de la resistencia a la compresión.
Tabla 5. Resultados del análisis de varianza de dos factores.

\begin{tabular}{cccccccc}
\hline $\begin{array}{c}\text { Origen } \\
\text { de la } \\
\text { variación }\end{array}$ & $\begin{array}{c}\text { Suma de } \\
\text { cuad. }\end{array}$ & $\begin{array}{c}\text { Grados } \\
\text { de } \\
\text { libertad }\end{array}$ & Prom. & $\begin{array}{c}\text { Valor de } \\
\text { Fisher } \\
\text { (F) }\end{array}$ & Prob. & $\begin{array}{c}\text { Valor de } \\
\text { Fisher } \\
\text { crítico } \\
\text { (F } \mathbf{c o s}\end{array}$ \\
$\begin{array}{c}\text { Condición } \\
\text { de Carga }\end{array}$ & 226 & 1 & 226 & 2.35 & 0.141 & 4.35 \\
$\begin{array}{c}\text { Condición } \\
\text { Física }\end{array}$ & 22079 & 1 & 22079 & 229.78 & $1.9 \times 10^{-12}$ & 4.35 \\
$\begin{array}{c}\text { Interacción } \\
\text { Pentro del }\end{array}$ & 0.0001 & 1 & 0.0001 & 0.000001 & 0.999 & 4.35 \\
$\begin{array}{c}\text { grupo } \\
\text { Total }\end{array}$ & 1922 & 20 & 96 & - & - & - \\
\hline
\end{tabular}

condiciones de carga (carga paralela y carga perpendicular). De esta forma en la Tabla 5, se observan los resultados del análisis de varianza de dos factores.

En la Tabla 5, se observa que en relación con la condición física de las probetas (saturada o seca) se obtuvo que Fn $=229.78$ es mayor que $\mathrm{Fcr}=4.35$; por lo tanto, sí existe diferencia significativa con una significancia del 5\%. De esta forma, se determina que las probetas saturadas con carga paralela a la veta son las que tienen la menor resistencia a la compresión con un valor promedio de $56.35 \mathrm{MPa}$.

\section{Discusión}

Aunque la norma de especificaciones para lajas ASTM C629-03 Standard Specification for Slate Dimension Stone [24] no tiene un valor específico para la resistencia a la compresión, se pudo comparar con otras especificaciones de materiales pétreos naturales. En la Tabla 6, se muestran los valores de la resistencia a la compresión mínima de mármoles, piedras calizas, serpentinos, travertinos, granitos y piedras con base de cuarzo.

Con base en la resistencia a la compresión dadas por las normas enlistadas en la Tabla 6, la laja tipo salmón procedente de San José de Gracia, Molcaxac, Puebla, México, que tuvo una resistencia a la compresión mínima de 56.35 MPa presenta niveles de resistencia a la compresión por arriba de las que se solicitan para el mármol, la piedra caliza, el travertino y la arenisca.

Además, si se compara la resistencia a la compresión de la laja tipo salmón con la resistencia a la compresión de diversos tipos de rocas internacionales mostradas en la Tabla 1, se puede decir que la laja tipo Salmón tiene una resistencia a la compresión por arriba del travertino tipo Atashkooh White, travertino tipo Plivit Jajce, de la piedra caliza tipo Mármol Royal 
Tabla 6. Comparación de la resistencia a la compresión de diferentes materiales pétreos naturales.

$\begin{array}{cc}\text { Norma } & \begin{array}{c}\text { Resistencia a la } \\ \text { Compresión (MPa) }\end{array} \\ \text { ASTM C503/C503M-15 } & 52 \\ \text { (Mármol) [25] } & 12 \text { (Baja densidad) } \\ \text { ASTM C568/C568M-10 (Piedra } & 28 \text { (Media densidad) } \\ \text { Caliza) [26] } & 55 \text { (Alta densidad) } \\ \text { ASTM C1526-08 (Serpentino) } & 69 \\ \text { [27] } & 34 \text { (Interiores) } \\ \text { ASTM C1527/C1527M-11 } & 52 \text { (Exteriores) } \\ \text { (Travertino) [28] } & 131 \\ \text { ASTM C615-03 (Granito) [29] } & 27.6 \text { (Arenisca) } \\ & 68.9 \text { (Arenisca } \\ \text { ASTM C616-03 (A base de } & \text { cuarzítica) } \\ \text { cuarzo) [30] } & 137.9 \text { (Cuarzita) }\end{array}$

Bronce y de la roca del afloramiento de Cojitambo; por lo tanto, también puede ser considerada como una roca para fines de exportación. También, al compararla con la resistencia a la compresión de diversos tipos de rocas nacionales mostradas en la Tabla 2, se puede argumentar que la laja tipo salmón tiene una mayor resistencia a la compresión que la toba riolítica y que las rocas calizas de Yucatán y de El Rosario Xochitiopan.

\section{Conclusión}

Como resultado de los ensayos de compresión uniaxial realizados, se obtuvo que las probetas saturadas con carga perpendicular a la veta tuvieron una resistencia a la compresión más baja en comparación con los demás grupos de probetas; por lo tanto, se puede decir que la laja tipo salmón procedente de San José de Gracia, Molcaxac, Puebla, México, tiene una resistencia a la compresión de $56.35 \mathrm{MPa}$. Habría que decir también que, la resistencia a la compresión es una medida de la resistencia a las cargas de aplastamiento; por ejemplo, si se construyera un muro de piedra, la piedra en la parte inferior tendría que soportar la carga de compresión del peso de las piedras de arriba [8].

Es importante reconocer que, la exigencia de los mercados por el cumplimiento de las propiedades mecánicas de la laja es cada vez mayor; por lo tanto, saber si la laja cumple con la resistencia a la compresión necesaria para una aplicación en particular, la pone en ventaja con respecto otros tipos de lajas. Por lo tanto, el conocer la resistencia a la compresión de una laja puede generar beneficios para: a) productores y proveedores, ya que les permite garantizar la calidad de su producto para que no existan reclamaciones por parte de los clientes; $b$ ) ingenieros y arquitectos, pueden disponer de esta propiedad para seleccionar la laja adecuada bajo un marco normativo; c) usuarios, puedan garantizar que su inversión va a cumplir con sus necesidades específicas.

Cabe mencionar que, la laja se suele utilizar como material para pisos y revestimientos de fachadas de casas y edificios, además de emplearse para la fabricación de piezas ornamentales; sin embargo, con un amplio conocimiento tanto de sus propiedades mecánicas como físicas, se podría extender su uso para otras aplicaciones. En este sentido, con base en los resultados del ensayo de compresión uniaxial y comparando estos resultados con otros materiales naturales utilizados en columnas como el mármol, el travertino y el granito, se puede argumentar que la laja tipo salmón procedente de San José de Gracia, Molcaxac, Puebla, México, es adecuada para usos tales como columnas para mesas, patas para sillas y pedestales para lavabos de casa-habitación.

Como comentario final, se considera que los ensayos que se deberán realizar a futuro a este material son los ensayos de abrasión y de fricción para determinar si la laja tipo Salmón se pueda considerar como material para pisos en situaciones de tráfico pesado; a su vez, se pretende realizar el ensayo de flexión para decidir si puede ser utilizada como peldaños de escaleras. Por lo que se refiere a estudio acelerados de degradación, otros ensayos relevantes son las pruebas de cristalización de sales para obtener la alteración que sufre esta laja en ambientes salinos o contaminados; además, realizar ensayos de helacidad para establecer la alteración sufrida en ambientes fríos. Incluso, realizar ensayos de humedad-sequedad para determinar el deterioro sufrido en condiciones de clima lluvioso.

\section{Agradecimientos}

Los autores reconocen a la empresa Mármoles Gómez por el apoyo brindado para procesar las probetas; también, reconocen al Instituto Tecnológico Superior de Tepexi de Rodríguez por el apoyo brindado para el financiamiento y de haber facilitado las instalaciones y equipos para la ejecución de las pruebas de compresión uniaxial.

\section{Referencias}

[1] M. Navarrete, W. Martínez, E. Alonso, C. Lara, A. Bedolla, H. Chávez, D. Delgado y J. Arteaga, "Caracterización de propiedades físico-mecánicas de rocas ígneas utilizadas en obras de infraestructura", ALCONPAT, vol. 3, núm. 2, pp. 132-142, mayo-agosto, 2013. 
[2] W. Orozco-Centeno, J. Branch, J. Jiménez-Builes, "Clasificación de rocas ígneas, sedimentarias y metamórficas en secciones delgadas a través programación estructurada", Boletín de Ciencias de la Tierra, vol. 36, pp. 5-9, diciembre, 2014.

[3] Standard Terminology Relating to Dimension Stone. ASTM C119-16, 2016.

[4] E. Tarbuck, F. Lutgens, Ciencias de la tierra. Una introducción a la geología física, 8a ed., Madrid, España: Pearson Education, 2005.

[5] L. Chia-Ming, F. Zhen-Yi, "Deformation characteristics of slate slopes associated with morphology and creep", Engineering Geology, vol. 178, pp. 132-154, agosto, 2014.

[6] Marble Institute of America, Dimension Stone Design Manual Version VIII: Slate, Ohio, USA: Marble Institute of America, 2016.

[7] T. Villanueva, La minería de la pizarra en Castilla y León, España, España: Sociedad de Investigación y Explotación Minera de Castilla y León (SIEMCALSA), 2010.

[8] Marble Institute of America, Dimension Stone Design Manual Version VIII: Stone testing, Ohio, USA: Marble Institute ofAmerica, 2016.

[9] R. Esbert, F. Alonso, J. Ordaz, "La petrofísica en la interpretación del deterioro y la conservación de la piedra de edificación", Trabajos de Geología, vol. 28, pp. 87-95, 2008. A. Jamshidi, M. Nikudel, M. Khamehchiyan, A. Zalooli,

[10] H. Yeganehfar. "Estimating the Mechanical Properties of Travertine Building Stones Due to Salt Crystallization Using Multivariate Regression Analysis", Journal of Sciences, vol. 28, núm. 3, pp. 231-241, 2017.

[11] L. Suárez, L. Calleja, I. Díez, V. Ruíz, A. Rodríguez, F. Alonso, "Características tecnológicas de las rocas ornamentales de Asturias", Trabajos de Geología, vol. 73, pp. 73-84, 2002.

[12] N. Ademovi, A. Kurtovi, "Stone and mortar in bridges from the Ottoman period in Bosnia and Herzegovina", GRAEVINAR, vol. 70, núm. 3, pp. 213-224, 2018.

[13] L. Torres, J. Poterico, "Propiedades físico-mecánicas de las rocas o minerales y su aplicación en el diseño de obras de ingeniería en el Callejón de Huaylas - 2013", Aporte Santiaguino, vol. 10, núm. 1, pp. 99-106, 2017.

[14] D. Rocha-Vargas, J. Becerra-Becerra, D. Benavente, J. Cañaveras, A. Costa, "Estudio preliminar de las características petrográficas, petrofísicas y comportamiento mecánico de rocas naturales tipo "Piedra Bogotana" y "Mármol Royal Bronce" utilizadas en construcciones patrimoniales y recientes en Colombia", UIS Ingenierías, vol. 18, núm. 3, pp. 203-222, 2019.

[15] R. Celi, D. Michelle, "Correlación entre la deformación y la resistencia a la compresión uniaxial en rocas", Trabajo de Grado, Escuela de Ingeniería en Minas, Facultad de Ciencia y Tecnología, Universidad del Azuay, Cuenca, Ecuador, 2019.

[16] L. Alonso, L. Espinoza, "Estudio de las propiedades de la roca caliza de Yucatán", Ingeniería, vol. 7, núm. 1, pp. 27-36, 2003.

[17] J. Sánchez, Inventario físico de los recursos minerales del municipio Tierra Blanca, Gto., Hidalgo, México: Consejo de Recursos Minerales, 2003

[18] H. Arriaga, Inventario físico de los recursos minerales de la carta La Flor de Jimulco G13-D46, México: Servicio Geológico Mexicano, 2012.

[19] A. Morales, T. Flores, M. Velasco, "Estudio de la resistencia a la compresión del travertino tipo Puebla procedente de Moralillo, Tepexi de Rodríguez, Puebla, México", Ingeniantes, año 5, vol. 1, núm. 2, pp. 18-25, noviembre, 2018.

[20] A. Morales-Tassinari, M. Velasco-Ordóñez y P. CruzOrtega, "Resistencia a la compresión de la roca caliza procedente de la cantera de la localidad de El Rosario Xochitiopan, Zacapala, Puebla, México", en XVII Congreso Nacional de Ingeniería Electromecánica y de Sistemas (CNIES 2018), Ciudad de México, México, 12-16 octubre, 2018.

[21] A. Morales-Tassinari, M. Azcaray-Rivera, M. Velasco, A. Servín-Martínez, J. Hernández-Zárate, "Caracterización físico-mecánica de dos travertinos de la región de Tepexi de Rodríguez, Puebla, México", en XXV Congreso Internacional Anual de la SOMIM, Mazatlán, Sinaloa, México, 18-20 septiembre, 2019.

[22] Standard Test Method for Compressive Strength. ASTM C170/C170M-16.2016.

[23] J. Hernández, P. Quinto, "Secado de medios porosos: una revisión a las teorías actualmente en uso", Científica, vol. 9, núm. 2, pp. 63-71, 2005.

[24] Standard Specification for Slate Dimension Stone. ASTM C529-03. 2004.

[25] Standard Specification for Marble Dimension Stone. ASTM C503/C503M-15.2015.

[26] Standard Specification for Limestone Dimension Stone. ASTM C568/C568M-10. 2010.

[27] Standard Specification for Serpentine Dimension Stone. ASTM C1526-08. 2014.

[28] Standard Specification for Travertine Dimension Stone. ASTM C1527/C1527M-11.2011.

[29] Standard Specification for Granite Dimension Stone. ASTM C615-03. 2003.

[30] Standard Specification for Quartz-Based Dimension Stone. ASTM C616-03. 2003. 
